\title{
MONOTONICITY PROPERTIES OF THE ZEROS OF BESSEL FUNCTIONS
}

\author{
ROGER C. McCANN' and E. R. LOVE
}

(Received 31 July 1979; revised 9 June 1981)

\begin{abstract}
Let $j_{\nu}$ denote the first positive zero of $J_{\nu}$. It is shown that $j_{\nu} /(\nu+\alpha)$ is a strictly decreasing function of $\nu$ for each positive $\alpha$ provided $\nu$ is sufficiently large. For each $\alpha$ lowe bounds $^{2}$ on $\nu$ are given to assure the monotoncity of $j_{v} /(\nu+\alpha)$. From this it is shown that $J_{v}>\nu+j_{0}$ for all $\nu>0$, which is both simpler and an improvement on the well known inequality $J_{\nu} \geqslant(\nu(\nu+2))^{1 / 2}$.
\end{abstract}

\section{Introduction}

The zeros of Bessel functions have been investigated in depth because of their relation to physical phenomena. For example, the transverse vibrations of a membrane in the form of a circular sector, which is clamped along its edges, are determined by the zeros of Bessel functions of the first kind. The study of the behavior of the zeros of Bessel functions dates back at least as far as an entry in Gauss' notebook dated October 16, 1797 (see the footnote on page 506 of [15]). Since then results concerning the interlacing of zeros of various orders, monotonicity of growth, asymptotic expansions, and algebraic bounding functions for the zeros have been established. Here we shall show that if $j_{\nu}$ denotes the first positive zero of the Bessel function of the first kind $J_{\nu}$, then for any $\alpha>0, j_{\nu} /(\nu+\alpha)$ is a strictly decreasing function whenever $\nu$ is sufficiently large. For each $\alpha>0$, a lower bound to $\nu$ which ensures the monotonicity of $j_{\nu} /(\nu+\alpha)$ is given. From this it is shown that $j_{\nu}>\nu+j_{0}$ for all $\nu>0$. This is an improvement on the well known inequality $j_{\nu} \geqslant(\nu(\nu+2))^{1 / 2}$.

\footnotetext{
I Department of Mathematics, Mississippi State University, Mississippi State, Mississippi 39762, U.S.A.

${ }^{2}$ Department of Mathematics, University of Melbourne, Parkville, Victoria, 3052

(1) Copyright Australian Mathematical Society 1982
} 


\section{Preliminary lemmas}

We begin by considering the eigenvalue problem

$$
\begin{gathered}
-\left(x y^{\prime}\right)^{\prime}+A^{2} x^{-1} y=\lambda x^{2 p-1} y \quad \text { for } a<x<1, \\
y(a)=y(1)=0,
\end{gathered}
$$

where $0<a<1,0<p$, and $A \equiv A(p)=1-\alpha \hat{p}$ on the intervai $\left(0, \alpha^{-1}\right)$; and $\alpha>0$. It is easily verified that the general solution of equation (1) is

$$
C_{1} J_{A / p}\left(\lambda^{1 / 2} x^{p} / p\right)+C_{2} Y_{A / p}\left(\lambda^{1 / 2} x^{p} / p\right)
$$

and that the eigenvalues $\lambda$ are the positive zeros of

$$
g(p, a, \lambda)=J_{A / p}\left(\lambda^{1 / 2} / p\right)-\frac{J_{A / p}\left(\lambda^{1 / 2} a^{p} / p\right)}{Y_{A / p}\left(\lambda^{1 / 2} a^{p} / p\right)} Y_{A / p}\left(\lambda^{1 / 2} / p\right) .
$$

Let $R[p, y, a]$ denote the Rayleigh quotient

$$
R[p, y, a]=\frac{\int_{a}^{1}\left\{\left(-x y^{\prime}\right)^{\prime}+A^{2} x^{-1} y\right\} y d x}{\int_{a}^{1} x^{2 p-1} y^{2} d x} .
$$

It is well known that the eigenvalues $\left\{\lambda_{n}(p, a)\right\}$ of (1), (2) can be obtained from the Rayleigh quotient [10, Sections 31 and 35]. Let $V(a)$ denote the linear space of all functions in $C^{2}([a, 1])$ which satisfy the boundary conditions (2). Then

$$
\lambda_{1}(p, a)=\min _{\substack{y \in V(a) \\ y \neq 0}} R[p, y, a] .
$$

Moreover, if $y_{1}$ is an eigenfunction of (1), (2) associated with $\lambda_{1}(p, a)$, then $\lambda_{1}(p, a)=R\left[p, y_{1}, a\right]$. Note that the eigenfunction $y_{1}$ may be chosen to be

$$
y_{1}(p, a, x)=J_{A / p}\left(\lambda_{Y}^{1 / 2} x^{p} / p\right)-\frac{J_{A / p}\left(\lambda^{1} /{ }^{2} a^{p} / p\right)}{Y_{A / p}\left(\lambda^{1 / 2} a^{p} / p\right)} Y_{A / p}\left(\lambda_{1}^{1 / 2} x^{p} / p\right),
$$

where $\lambda_{1} \equiv \lambda_{1}(p, a)$.

LEMma 1. If $0<B<C$, then there is an $x_{0} \in(0, B)$ such that $\left|Y_{t}(x)\right| \leqslant\left|Y_{t}(z)\right|$ whenever $0<z \leqslant x_{0}, z \leqslant x$, and $B \leqslant t \leqslant C$.

Proof. The integral representation, for $x>0$,

$$
Y_{t}(x)=\pi^{-1} \int_{0}^{\pi} \sin (x \sin \theta-t \theta) d \theta-\pi^{-1} \int_{0}^{\infty}\left(e^{t s}+e^{-t s} \cos t \pi\right) e^{-x \sinh s} d s
$$


is given on page 178 of [15]. If $B \leqslant x$ and $0<t \leqslant C$, then

$$
\begin{aligned}
\left|Y_{t}(x)\right| & \leqslant 1+\pi^{-1} \int_{0}^{\infty} 2 e^{t s} e^{-x \sinh s} d s \\
& \leqslant 1+2 \pi^{-1} \int_{0}^{\infty} e^{c s-B \sinh s} d s
\end{aligned}
$$

the latter integral being convergent. Thus there is an $M$ such that

$$
\left|Y_{\imath}(x)\right| \leqslant M
$$

whenever $B \leqslant x$ and $B \leqslant t \leqslant C$.

Let $y_{t}$ and $j_{t}^{\prime}$ denote the least positive zeros of $Y_{t}$ and $J_{t}^{\prime}$. On pages 485 and 487 of [15] it is shown that $t<j_{t}^{\prime}$ and that $Y_{t}(x)^{2}$ is strictly decreasing on $0<x<j_{t}^{\prime}$ provided $0<t$. Thus $\left|Y_{t}(x)\right|$ is decreasing and positive on $0<x \leqslant t$ if $0<t$. In particular,

$$
\left.\begin{array}{l}
\left|Y_{t}(x)\right| \text { is a decreasing function of } x \\
\text { on } 0<x \leqslant B \text { whenever } B \leqslant t \leqslant C, \\
\text { and }\left|Y_{t}(x)\right|>0 \text { for these values of } x \text { and } t .
\end{array}\right\}
$$

For positive integral $n$, let $f_{n}(t)=1 /\left|Y_{t}(B / n)\right|$. By (7), these functions form a decreasing sequence of continuous functions of $t$ on $[B, C]$. Moreover, $f_{n}(t) \rightarrow 0$ as $n \rightarrow \infty$. By Dini's Theorem (page 140 of [13]) this convergence is uniform. Hence there is a positive integer $n_{0}$ such that $\left|f_{n}(t)\right|<1 / M$ whenever $n_{0}<n$ and $t \in[B, C]$. Set $x_{0}=B /\left(n_{0}+1\right)$. Then

$$
M<\left|Y_{t}\left(x_{0}\right)\right| \text { whenever } B \leqslant t \leqslant C \text {. }
$$

Suppose $0<z<x_{0}, z \leqslant x$, and $B \leqslant t \leqslant C$. If $B \leqslant x$, then (6), (7) and (8) give

$$
\left|Y_{t}(x)\right| \leqslant M<\left|Y_{t}\left(x_{0}\right)\right| \leqslant\left|Y_{t}(z)\right|
$$

so that $\left|Y_{t}(x)\right| \leqslant\left|Y_{t}(z)\right|$. If $x<B$ this last inequality follows from (7) only.

LEMMA 2. Let $\alpha, b, c, d$ be positive constants such that $0<b<c<\alpha^{-1}$. Set $t=p^{-1}-\alpha$ and

$$
g(p, a, x, \lambda)=J_{t}\left(\lambda^{1 / 2} x^{p} / p\right)-\frac{J_{t}\left(\lambda^{1 / 2} a^{p} / p\right)}{Y_{t}\left(\lambda^{1 / 2} a^{p} / p\right)} Y_{t}\left(\lambda^{1 / 2} x^{p} / p\right) .
$$

Then there are positive constants $a_{0}<\frac{1}{2}, k_{0}$ and $k$ such that

$$
\left|g(p, a, x, \lambda)-J_{t}\left(\lambda^{1 / 2} x^{p} / p\right)\right|<k_{0} a^{k}
$$

whenever $0<a \leqslant a_{0}, a \leqslant x, b \leqslant p \leqslant c$, and $0<\lambda \leqslant d$. 
Proof. Set $B=c^{-1}-\alpha$ and $C=b^{-1}-\alpha$ so that $0<B<C$. Then $t \in[B, C]$ if and only if $p \in[b, c]$. With $x_{0}$ as in Lemma 1 , choose $a_{0} \in\left(0, \frac{1}{2}\right)$ so that $d^{1 / 2} a_{0}^{p} / b<x_{0}$. Then $0<\lambda^{1 / 2} a^{p} / p<x_{0}$ whenever $0<a \leqslant a_{0}, b \leqslant p \leqslant c$, and $0<\lambda \leqslant d$. Replacing $z$ and $x$ in Lemma 1 by $\lambda^{1 / 2} a^{p} / p$ and $\lambda^{1 / 2} x^{p} / p$ respectively yields

$$
\left|Y_{t}\left(\lambda^{1 / 2} x^{p} / p\right)\right| \leqslant\left|Y_{t}\left(\lambda^{1 / 2} a^{p} / p\right)\right|
$$

whenever $0<a \leqslant a_{0}, 0<a \leqslant x, b \leqslant p \leqslant c$, and $0<\lambda \leqslant d$. By (9), (10), and inequality (1) on page 49 of [15] we have

$$
\begin{aligned}
\left|g(p, a, x, \lambda)-J_{t}\left(\lambda^{1 / 2} x^{p} / p\right)\right| & \leqslant\left|J_{t}\left(\lambda^{1 / 2} a^{p} / p\right)\right| \\
& \leqslant \frac{\left(\frac{1}{2} \lambda^{1 / 2} a^{p} / p\right)^{t}}{\Gamma(1+t)} \\
& \leqslant \frac{\left(\frac{1}{2} d^{1 / 2} / b\right)^{t}}{\Gamma(1+t)} a^{1-\alpha p} \\
& \leqslant \max \left\{1,\left(\frac{1}{2} d^{1 / 2} / b\right)^{C}\right\} m^{-1} a^{1-\alpha c}
\end{aligned}
$$

where $0<m$ is a lower bound for $\Gamma(1+t)$ on $[B, C]$. Since $c<\alpha^{-1}$ the inequality in (11) is the desired inequality.

Lemma 3. Let $\alpha, b, c$ be positive constants such that $0<b<c<\alpha^{-1}$. Set $t=p^{-1}-\alpha$ and let $\lambda=\lambda_{1} \equiv \lambda_{1}(p, a)$ denote the least positive root of

$$
g(p, a, 1, \lambda)=0
$$

where $g$ is defined by (9). Set $y_{1}(p, a, x)=g\left(p, a, x, \lambda_{1}\right)$. Then there are positive constants $a_{0}<\frac{1}{2}, k_{1}$ and $k$ such that

$$
\left|y_{1}(p, a, x)-J_{t}\left(\lambda_{1}^{1 / 2} x^{p} / p\right)\right|<k_{1} a^{k}
$$

whenever $0<a \leqslant a_{0}, a \leqslant x$ and $b \leqslant p \leqslant c$. Moreover, $\lambda_{1}^{1 / 2}(p, a) / p$ converges to a zero of $J_{t}$ as $a \rightarrow 0+$.

Proof. Assume by analogy with the results on pages 409 and 419 of [2] that

(i) $\lambda_{1}(p, a)$ is a nondecreasing function of $a$ in $0<a<1$,

(ii) $\lambda_{1}(p, a)$ is a continuous function of $p$ for fixed $a$.

By (ii), $\lambda_{1}\left(p, \frac{1}{2}\right)$ is continuous on $b \leqslant p \leqslant c$ and therefore bounded, say by $N$ : $0<\lambda_{1}\left(p, \frac{1}{2}\right) \leqslant N$ whenever $b \leqslant p \leqslant c$. By (i), $0<\lambda_{1}(p, a) \leqslant \lambda_{1}\left(p, \frac{1}{2}\right) \leqslant N$ whenever $0<a \leqslant \frac{1}{2}$ and $b \leqslant p \leqslant c$.

Lemma 2 with $d$ and $\lambda$ replaced by $N$ and $\lambda_{1}=\lambda_{1}(p, a)$ respectively now gives the desired inequality (12). 
With $x=1$ this inequality becomes

$$
\left|J_{t}\left(\lambda_{1}^{1 / 2}(p, a) / p\right)\right|<k_{1} a^{k} .
$$

Since $\lambda_{1}(p, a)$ is a nondecreasing function of $a$ there is a nonnegative number $l$ such that $\lambda_{1}(p, a) \rightarrow l$ as $a \rightarrow 0+$. If we now let $a \rightarrow 0+$ the above inequality yields $J_{t}\left(l^{1 / 2} / p\right)=0$ so that $l^{1 / 2} / p$ is a zero of $J_{t}$. The last sentence of the lemma follows.

LemMA 4. With the notation of Lemma $3, \lambda_{1}^{1 / 2}(p, a) \rightarrow p j_{t}$ as $a \rightarrow 0+$, for $b \leqslant p \leqslant c$.

PRoof. Set $\lambda_{1}=\lambda_{1}(p, a)$. Since $J_{t}\left(\lambda_{1}^{1} /{ }^{2} x^{p} / p\right)$ and $y_{1}(p, a, x)$ are solutions of equation (1) with $\lambda=\lambda_{1}$, consecutive zeros on $(0, \infty)$ of either function are separated by a zero of the other, by the Sturm comparison theorem. Thus, between $a$ and 1 (zeros of $y_{1}(p, a, x)$ ) there is a zero of $J_{t}\left(\lambda_{1}^{1 / 2} x^{p} / p\right)$. Therefore the least positive zero $\left(\lambda_{1}^{-1 / 2} p j_{t}\right)^{1 / p}$ of $J_{t}\left(\lambda_{1}^{1 / 2} x^{p} / p\right)$ is in the interval $(0,1)$. Since $\lambda_{1}(p, a)$ is nondecreasing in $a$ (for fixed $\left.p\right)$,

$$
0<\lambda_{1}^{-1 / 2}\left(p, \frac{1}{2}\right) p j_{t} \leqslant \lambda_{1}^{-1 / 2}(p, a) p j_{t}
$$

whenever $0<a<\frac{1}{2}$. Thus, if $0<a<\min \left\{\frac{1}{2},\left(\lambda_{1}^{-1 / 2}\left(p, \frac{1}{2}\right) p j_{t}\right)^{1 / p}\right\}$,

$$
a<\left(\lambda_{1}(p, a)^{-1 / 2} p j_{t}\right)^{1 / p}<1 ;
$$

that is, this inequality holds for $a$ sufficiently small.

Let $j_{t, 2}$ be the second positive zero of $J_{t}$. By Sturm's theorem again, between the first two positive zeros $\left(\lambda_{1}^{-1 / 2} p j_{t}\right)^{1 / p}$ and $\left(\lambda_{1}^{-1 / 2} p j_{t, 2}\right)^{1 / p}$ of $J_{t}\left(\lambda_{1}^{1 / 2} x^{p} / p\right)$ there is a zero of $y_{1}(p, a, x)$. It is known (see page 452 of [2]) that an eigenfunction corresponding to the smallest eigenvalue of a Sturm-Liouville system, such as in equations (1) and (2), does not change sign on the interval whose endpoints are given in the boundary conditions. Thus there are no zeros of $y_{1}(p, a, x)$ between $a$ and 1 , so that

$$
\left(\lambda_{1}^{-1 / 2} p j_{t}\right)^{1 / p}<1<\left(\lambda_{1}^{-1 / 2} p j_{t, 2}\right)^{1 / p},
$$

or equivalently

$$
p j_{t}<\lambda^{1 / 2}<p j_{t, 2} \text {. }
$$

Since $\lambda_{1}$ is a nondecreasing function of $a$ (for fixed $p$ ) we have

$$
p j_{t} \leqslant \lim _{a \rightarrow 0+} \lambda_{1}^{1 / 2}<p j_{t, 2} \text {. }
$$

The desired result now follows directly from Lemma 3.

LEMMA 5. With the notation of Lemma $3, \lambda_{1}^{1 / 2}(p, a) \rightarrow p j_{t}$ as $a \rightarrow 0+$ uniformly on $b \leqslant p \leqslant c$. 
Proof. For clarity we denote $t$, that is, $p^{-1}-\alpha$, by $t(p)$. Let $\left\{a_{n}\right\}$ be a decreasing sequence tending to 0 as $n \rightarrow \infty$. Then $\lambda^{1 / 2}\left(p, a_{n}\right)$ is nonincreasing, by (i) of Lemma 3; and by Lemma 4

$$
\lambda_{1}^{1 / 2}\left(p, a_{n}\right) \rightarrow p j_{t(p)} \text { as } n \rightarrow \infty
$$

for $b \leqslant p \leqslant c$. For these values of $p, t(p)$ is decreasing, so

$$
t(p) \geqslant t(c)=c^{-1}-\alpha>0,
$$

since $0<b<c<\alpha^{-1}$. Thus $p j_{t(p)}$ is a continuous function of $p$. But $\lambda_{1}^{1 / 2}\left(p, a_{n}\right)$ is also continuous, for each fixed $n$, by (ii) of Lemma 3. Dini's Theorem (page 140 of [13]) accordingly shows that the convergence of the sequence $\lambda^{1} /{ }^{2}\left(p, a_{n}\right)$ is uniform on $b \leqslant p \leqslant c$.

The stated conclusion now follows since $\lambda^{1} /{ }^{2}(p, a)$ is a nondecreasing function of $a$.

LEMMA 6. Under the hypotheses of Lemma 3, together with $0<\xi<1$,

$$
y_{1}^{2}(p, a, x) \rightarrow J_{t}^{2}\left(j_{t} x^{p}\right) \text { as } a \rightarrow 0+
$$

uniformly for $\xi \leqslant x \leqslant 1$ and $b \leqslant p \leqslant c$.

Proof. As in Lemma 2, let $B=c^{-1}-\alpha$ and $C=b^{-1}-\alpha$. Since $1 / p$ is bounded on $b \leqslant p \leqslant c$, Lemma 5 gives that $\lambda^{1} /{ }^{2}(p, a) / p \rightarrow j_{t}$ as $a \rightarrow 0+$ uniformly on $b \leqslant p \leqslant c$. So there is an $a^{\prime}>0$ such that

$$
2 j_{C}>j_{C}+j_{t}>\lambda_{1}^{1 / 2} / p>j_{t}-\frac{1}{2} j_{B} \geqslant \frac{1}{2} j_{B}
$$

whenever $0<a<a^{\prime}$ and $b \leqslant p \leqslant c$, since then $B \leqslant t \leqslant C$. For $\xi \leqslant x \leqslant 1$ we thus have

$$
\frac{1}{2} j_{B} \xi^{c}<\lambda^{1 / 2} x^{p} / p<2 j_{C}
$$

whenever $0<a<a^{\prime}$ and $b \leqslant p \leqslant c$. Since $J_{t}(u)$ is uniformly continuous on $B \leqslant t \leqslant C, \frac{1}{2} j_{B} \xi^{c} \leqslant u \leqslant 2 j_{C}$,

$$
J_{t}\left(\lambda_{1}^{1 / 2} x^{p} / p\right) \rightarrow J_{t}\left(j_{t} x^{p}\right)
$$

as $a \rightarrow 0+$ uniformly for $b \leqslant p \leqslant c$ and $\xi \leqslant x \leqslant 1$. Also, by Lemma 3,

$$
y_{1}(p, a, x)-J_{t}\left(\lambda_{1}^{1} /{ }^{2} x^{p} / p\right) \rightarrow 0
$$

as $a \rightarrow 0+$ uniformly for $b \leqslant p \leqslant c$ and $\xi \leqslant x \leqslant 1$. Adding (13) and (14) gives

$$
y_{1}(p, a, x) \rightarrow J_{t}\left(j_{t} x^{p}\right)
$$

as $a \rightarrow 0+$ uniformly for $b \leqslant p \leqslant c$ and $\xi \leqslant x \leqslant 1$.

It is easily proved that if $f_{n}(x) \rightarrow f(x)$ uniformly for $x \in S$, as $n \rightarrow \infty$, and $f(x)$ is bounded on the set $S$, then $f_{n}^{2}(x) \rightarrow f^{2}(x)$ uniformly for $x \in S$. It is known that ([1], page 362) $\left|J_{\nu}(z)\right| \leqslant 1$ for all $\nu \geqslant 0$ and $z \geqslant 0$. The desired result follows. 


\section{The function $K(\nu)$}

LEMMa 7. For $\nu>0$ let $j_{\nu}$ and $j_{\nu}^{\prime}$ denote the least positive zeros of $J_{\nu}$ and $J_{\nu}^{\prime}$ respectively. Let $K(\nu)$ denote the least positive integer $m$ such that $j_{\nu}<j_{\nu+m}^{\prime}$. Then $K(\nu)$ exists, and

(i) for fixed $\lambda \geqslant \frac{1}{4}$ such that $K(\lambda) \geqslant 3, K(\nu) \geqslant 3$ for all $\nu \geqslant \lambda$;

(ii) for fixed $\lambda \geqslant 5$ such that $K(\lambda) \geqslant 4, K(\nu) \geqslant 4$ for all $\nu \geqslant \lambda$.

Proof. Existence of $K(\nu)$ for all $\nu>0$ follows from the facts that $j_{\nu}>j_{\nu}^{\prime}$ and $j_{\mu}^{\prime}>\mu$ for all $\mu>0$ (see [15] page 485).

The proofs of (i) and (ii) are similar; we will prove (ii). We do not prove here the existence of a $\lambda$ such that $\lambda \geqslant 5$ and $K(\lambda) \geqslant 4$; for that see Lemma 8 .

Suppose that $\lambda \geqslant 5, K(\lambda) \geqslant 4, \nu>\lambda$ and $K(\nu)<4$, and seek a contradiction. Let $\mu$ be the lower bound of numbers $t>\lambda$ such that $K(t)<4$; then $\lambda \leqslant \mu \leqslant \nu$. There is a sequence $t_{n} \rightarrow \mu+$ such that $K\left(t_{n}\right)<4$, and so $K\left(t_{n}\right) \leqslant 3$ since $K$ is integer-valued. Hence

$$
j_{t_{n}}<j_{t_{n}}^{\prime}+K\left(t_{n}\right) \leqslant j_{t_{n}+3}^{\prime},
$$

using the increasing property of $j_{v}^{\prime}$ (see [15] page 510). Thus $j_{t}-j_{t+3}^{\prime}$, which is a continuous function of $t$, has nonpositive limit as $t \rightarrow \mu+$.

If $\mu>\lambda, K(t) \geqslant 4$ for $\lambda \leqslant t<\mu$, and so $K(t)>3$. By definition of $K, j_{t} \geqslant j_{t+3}^{\prime}$; thus the continuous function $j_{t}-j_{t+3}^{\prime}$ has nonnegative limit as $t \rightarrow \mu-$. With the conclusion of the previous paragraph this gives that $j_{\mu}-j_{\mu+3}^{\prime}=0$. The function $j_{t}-j_{t+3}^{\prime}$ is also differentiable, and so

$$
\left.\frac{d}{d t}\left(j_{t}-j_{t+3}^{\prime}\right)\right|_{t=\mu} \leqslant 0 .
$$

Similarly, if $\mu=\lambda$ we have since $K(\lambda)>3$ that $j_{\lambda} \geqslant j_{\lambda+3}^{\prime}$. And by a previous paragraph the continuous function $j_{t}-j_{t+3}^{\prime}$ has nonpositive limit as $t \rightarrow \lambda+$. It follows that it vanishes at $t=\lambda$. Further, since $t_{n} \rightarrow \lambda+$ and $j_{t_{n}}-j_{t_{n}+3}^{\prime}<0$, its derivative is nonpositive at $t=\lambda$.

By the two preceding paragraphs we have constructed $\mu \geqslant \lambda$, and so $\mu \geqslant 5$, such that

$$
j_{\mu}-j_{\mu+3}^{\prime}=0 \text { and }\left.\frac{d}{d t}\left(j_{t}-j_{t+3}^{\prime}\right)\right|_{t=\mu} \leqslant 0 .
$$

The rest of the proof develops a contradiction of this.

Using the standard identity $x J_{t}^{\prime}(x)+t J_{t}(x)=x J_{t-1}(x)$ once and the identity $J_{t-1}(x)+J_{t+1}(x)=(2 t / x) J_{t}(x)$ twice, we find that

$$
\mu J_{\mu+3}^{\prime}\left(j_{\mu}\right)=\left\{(3 \mu+5)-4(\mu+1)(\mu+2)(\mu+3) / j_{\mu}^{2}\right\} J_{\mu+1}\left(j_{\mu}\right)
$$


The left side vanishes since $j_{\mu}=j_{\mu+3}^{\prime} ;$ but $J_{\mu+1}\left(j_{\mu}\right) \neq 0$ since $j_{\mu}<j_{\mu+1}$. Consequently

$$
j_{\mu}^{2}=4(\mu+1)(\mu+2)(\mu+3) /(3 \mu+5) .
$$

We shall also need the formula

$$
\frac{d j_{\nu}^{\prime}}{d \nu}=2 j_{\nu}^{\prime} \int_{0}^{\infty} \frac{j_{\nu}^{\prime 2} \cosh 2 t-\nu^{2}}{j_{\nu}^{\prime 2}-\nu^{2}} K_{0}\left(2 j_{\nu}^{\prime} \sinh t\right) e^{-2 v t} d t
$$

from the Note on page 510 of [15]; it holds for all $\nu>0$. Here $K_{0}$ is the modified Bessel function of the second kind of order 0 .

Consider for $t \geqslant 0$ the function

$$
g(t)=\left\{j_{\mu+3}^{\prime 2} \cosh 2 t-(\mu+3)^{2}\right\}-\left\{j_{\mu+3}^{\prime 2}-(\mu+3)^{2}\right\} e^{6 t} .
$$

To prove this decreasing we have, since $j_{\mu+3}^{\prime}=j_{\mu}$,

$$
\begin{aligned}
g^{\prime}(t) & =2 j_{\mu}^{2} \sinh 2 t-6\left\{j_{\mu}^{2}-(\mu+3)^{2}\right\} e^{6 t} \\
& =6 e^{6 t}\left\{(\mu+3)^{2}-\left(1-\frac{1}{3} e^{-6 t} \sinh 2 t\right) j_{\mu}^{2}\right\} .
\end{aligned}
$$

Now $e^{-6 t} \sinh 2 t$ is maximum when $\tanh 2 t=\frac{1}{3}, \sinh 2 t=1 / 2 \sqrt{2}, e^{-2 t}=1 / \sqrt{2}$ and $e^{-6 t} \sinh 2 t=\frac{1}{8}$. So

$$
\begin{aligned}
g^{\prime}(t) & \leqslant 6 e^{6 t}\left\{(\mu+3)^{2}-(23 / 24) j_{\mu}^{2}\right\} \\
& =e^{6 t}(3 \mu+5)^{-1}(\mu+3)\{6(\mu+3)(3 \mu+5)-23(\mu+1)(\mu+2)\} \\
& =-e^{6 t}(3 \mu+5)^{-1}(\mu+3)\left(5 \mu^{2}-15 \mu-44\right) .
\end{aligned}
$$

Since $\mu \geqslant 5$, this is negative and so $g(t)$ is decreasing.

Now $g(0)=0$; consequently $g(t)<0$ for $t>0$, and so

$$
\begin{aligned}
\frac{d j_{\mu+3}^{\prime}}{d \mu} & <2 j_{\mu+3}^{\prime} \int_{0}^{\infty} e^{6 t} K_{0}\left(2 j_{\mu+3}^{\prime} \sinh t\right) e^{-2(\mu+3) t} d t \\
& =2 j_{\mu} \int_{0}^{\infty} K_{0}\left(2 j_{\mu} \sinh t\right) e^{-2 \mu t} d t=\frac{d j_{\mu}}{d \mu}
\end{aligned}
$$

using (3) on page 508 of [15]. This provides the desired contradiction.

Lemma 8. Let $K(\nu)$ be as in Lemma 7. Then

(i) $K(\nu) \rightarrow \infty$ as $\nu \rightarrow \infty$,

(ii) if $m$ is a positive integer, then $J_{\nu+m}^{\prime}\left(j_{\nu}\right)>0$ if and only if $K(\nu) \leqslant m$,

(iii) $K(\nu) \geqslant 2$ if $\nu>0$,

(iv) $K(\nu) \geqslant 3$ if $\nu \geqslant 3.5$,

(v) $K(\nu) \geqslant 4$ if $\nu \geqslant 18.5$. 
PROOF. (i) The asymptotic expansions

$$
j_{t}=t+c t^{1 / 3}+O\left(t^{-1 / 3}\right), \quad j_{t}^{\prime}=t+c^{\prime} t^{1 / 3}+O\left(t^{-1 / 3}\right)
$$

when $t \rightarrow \infty$, where $c=1.8557571 \ldots$ and $c^{\prime}=0.8086165 \ldots$, may be found in [14] or on page 371 of [1]. They give

$$
0<j_{\nu+K(\nu)}^{\prime}-j_{\nu}=K(\nu)+c^{\prime}(\nu+K(\nu))^{1 / 3}-c \nu^{1 / 3}+O\left(\nu^{-1 / 3}\right)
$$

as $\nu \rightarrow \infty$, since $(\nu+K(\nu))^{-1 / 3}<\nu^{-1 / 3}$.

Suppose that $K(\nu) \nrightarrow \infty$ as $\nu \rightarrow \infty$. Then there is $B$ such that $K(\nu) \leqslant B$ for arbitrarily large $\nu$; and so $K\left(\nu_{n}\right) \leqslant B$ for a sequence $\nu_{n} \rightarrow \infty$. Therefore, as $n \rightarrow \infty$,

$$
0<B+c^{\prime}\left(\nu_{n}+B\right)^{1 / 3}-c \nu_{n}^{1 / 3}+O\left(\nu_{n}^{-1 / 3}\right) .
$$

But the right side tends to $-\infty$ since $c>c^{\prime}>0$. This contradiction proves (i).

(ii) Suppose that $K(\nu) \leqslant m$. Using the inequality $\nu<j_{v}^{\prime}$ and the Note on page 510 of [15], we find that $j_{\nu}^{\prime}$ is an increasing function of $\nu$ for $\nu>0$. Since $\nu+K(\nu) \leqslant \nu+m$ we have

$$
j_{\nu}<j_{\nu+K(\nu)}^{\prime} \leqslant j_{\nu+m}^{\prime} .
$$

Since $J_{t}^{\prime}(x)>0$ for all $t>0$ and positive $x$ sufficiently small, $J_{t}^{\prime}(x)>0$ for $0<x<j_{t}^{\prime}$; consequently

$$
J_{\nu+m}^{\prime}(x)>0 \text { for } 0<x<j_{\nu+m}^{\prime} .
$$

In particular,

$$
J_{\nu+m}^{\prime}\left(j_{\nu}\right)>0 .
$$

Conversely, suppose this last inequality holds. Then the graph of $J_{\nu+m}^{\prime}$ shows that either $j_{v}<j_{v+m}^{\prime}$ or $j_{v+m, 2}^{\prime}<j_{v}$, where $j_{t, 2}^{\prime}$ is the second positive zero of $J_{t}^{\prime}$. The graph of $J_{t}$ shows that $j_{t}<j_{t, 2}^{\prime}$; and, by page 508 of [15], $j_{t}$ is increasing for $t>0$. Hence $j_{v}<j_{v+m} \leqslant j_{v+m, 2}^{\prime}$, which rules out the second alternative; and so $j_{v}<j_{v+m}^{\prime}$. Since $m$ is a positive integer, the definition of $K(\nu)$ now gives that $K(\nu) \leqslant m$, which completes the proof of (ii).

(iii) Using the recurrence formula $J_{t-1}(x)-J_{t+1}(x)=2 J_{t}^{\prime}(x)$ with $t=\nu+1$ and $x=j_{\nu}$ we have $-J_{\nu+2}\left(j_{v}\right)=2 J_{\nu+1}^{\prime}\left(j_{\nu}\right)$. Since $\nu>0$ and $0<j_{\nu}<j_{\nu+2}$, this gives

$$
2 J_{\nu+1}^{\prime}\left(j_{\nu}\right)=-J_{\nu+2}\left(j_{\nu}\right)<0 .
$$

Hence $j_{v+1}^{\prime}<j_{\nu}$. This proves that $K(\nu) \geqslant 2$, giving (iii).

(iv) and (v). Using the table on page 321 of [11],

$$
j_{5.5}^{\prime}<6.96<j_{3.5}, \quad j_{21.5}^{\prime}<23.78<j_{18.5} .
$$

Thus $K(3.5)>2$, from which Lemma 7 (i) gives that $K(\nu) \geqslant 3$ for all $\nu \geqslant 3.5$, proving (iv). Similarly $K(18.5)>3$, and Lemma 7 (ii) gives (v). 


\section{The integral $I(p)$}

The next four lemmas are to assist in showing that the Rayleigh quotient is an increasing function of $p$ whenever $p$ is sufficiently small. From this we shall be able to derive the desired results.

LEMMA 9. For $\alpha$ a positive constant, and $p \in\left(0, \alpha^{-1}\right)$, set $A(p)=1-\approx p$ and

$$
I=I(p)=p j_{v(p)}^{2} \int_{0}^{1}\left\{A^{\prime}(p)-A(p) \ln x\right\} x^{2 p-1} J_{v(p)}^{2}\left(j_{\nu(p)} x^{p}\right) d x .
$$

Then

$$
I(p)=\sum_{n=1}^{\infty}((\nu-\alpha) n-\alpha \nu) J_{\nu+n}^{2}(j),
$$

where $\nu$ and $j$ stand for $\nu(p)=A(p) / p=p^{-1}-\alpha$ and $j_{\nu(p)}$ respectively.

Proof. We begin by noting that $\nu>0$. If we change variables according to $t=j x^{p}$, integrate by parts, and use the asymptotic expansion, for small $z$, $J_{\nu}(z) \sim\left(2^{\nu} \Gamma(\nu+1)\right)^{-1} z^{\nu}$, we find that

$$
\begin{aligned}
I & =-\alpha \int_{0}^{j} t J_{\nu}^{2}(t) d t-\nu \int_{0}^{j}\left(\ln \frac{t}{j}\right) J_{\nu}^{2}(t) d t \\
& =-\alpha \int_{0}^{j} t J_{\nu}^{2}(t) d t+\nu \int_{0}^{j} t^{-1} \int_{0}^{t} s J_{\nu}^{2}(s) d s d t .
\end{aligned}
$$

Using the well known identity

$$
J_{\nu-1}(z)+J_{\nu+1}(z)=2 \nu z^{-1} J_{\nu}(z),
$$

we have $J_{v-1}(j)=-J_{v+1}(j)$. On page 135 of [15] it is shown that for $\omega>0$

$$
\int_{0}^{t} s J_{\omega}^{2}(s) d s=\frac{1}{2} t^{2}\left\{J_{\omega}^{2}(t)-J_{\omega-1}(t) J_{\omega+1}(t)\right\}
$$

With $t$ replaced by $j$, and $\omega$ successively replaced by $\nu+1, \nu$ and $\nu-1$, we find that

$$
\int_{0}^{J} J_{\nu}^{2}(t) d t=\int_{0}^{j} t J_{\nu-1}^{2}(t) d t=\int_{0}^{J} J_{\nu+1}^{2}(t) d t
$$

Using the recurrence formula (15) it is easy to verify that

$$
J_{\nu-1}(t) J_{\nu+1}(t)=-\frac{1}{2} J_{\nu-1}^{2}(t)-\frac{1}{2} J_{\nu+1}^{2}(t)+2 \nu^{2} t^{-2} J_{\nu}^{2}(t) .
$$


The identities (16), (17), and (18) enable us to write $I$ as

$$
\begin{aligned}
I & =-\alpha \int_{0}^{j} t J_{\nu}^{2}(t) d t+(\nu / 2) \int_{0}^{j} t\left\{J_{\nu}^{2}(t)-J_{\nu-1}(t) J_{\nu+1}(t)\right\} d t \\
& =(\nu / 2-\alpha) \int_{0}^{j} t J_{\nu}^{2}(t) d t+(\nu / 4) \int_{0}^{j} t\left\{J_{\nu-1}^{2}(t)+J_{\nu+1}^{2}(t)-4 \nu^{2} t^{-2} J_{\nu}^{2}(t)\right\} d t \\
& =\frac{1}{2}(\nu-\alpha) \int_{0}^{j} t J_{\nu}^{2}(t) d t+\frac{1}{2}(\nu-\alpha) \int_{0}^{j} J_{\nu+1}^{2}(t) d t-\nu^{3} \int_{0}^{j} t^{-1} J_{\nu}^{2}(t) d t .
\end{aligned}
$$

On pages 151 and 152 of [15] it is shown that

$$
\int_{0}^{z} U_{\omega-1}^{2}(t) d t=2 \sum_{n=0}^{\infty}(\omega+2 n) J_{\omega+2 n}^{2}(z)
$$

and

$$
2 \nu \int_{0}^{z} t^{-1} J_{\nu}^{2}(t) d t=J_{\nu}^{2}(z)+2 \sum_{n=1}^{\infty} J_{v+n}^{2}(z)
$$

These identities enable us to write $I$ as

$$
\begin{aligned}
I & =\sum_{n=1}^{\infty}(\nu-\alpha)(\nu+n) J_{\nu+n}^{2}(j)-\nu^{2} \sum_{n=1}^{\infty} J_{\nu+n}^{2}(j) \\
& =\sum_{n=1}^{\infty}((\nu-\alpha) n-\alpha \nu) J_{\nu+n}^{2}(j) .
\end{aligned}
$$

LEMMA 10. I $(p)$, defined in Lemma 9, is positive whenever $p$ is sufficiently small; that is, whenever $\nu(p)=p^{-1}-\alpha$ is sufficiently large. Specifically, $I(p)>0$ whenever $\nu>\alpha$ and $K(\nu)>2 \alpha \nu /(\nu-\alpha)$.

Proof. We will do the proof for the case $K(\nu)$ even; when $K(\nu)$ is odd the proof is analogous. We begin by noting that $0<J_{v+1}(j)$ and $0<J_{\nu+2}(j)$ since $j=j_{\nu}<j_{\nu+1}<j_{\nu+2}$.

For integers $n \geqslant K(\nu)$, with $\nu>\alpha$ and $K(\nu)>2 \alpha \nu /(\nu-\alpha)$,

$$
(\nu-\alpha) n-\alpha \nu \geqslant(\nu-\alpha) K(\nu)-\alpha \nu \geqslant 2 \alpha \nu-\alpha \nu=\alpha \nu>0 .
$$

Hence

$$
I(p)>\sum_{n=1}^{K(\nu)}((\nu-\alpha) n-\alpha \nu) J_{\nu+n}^{2}(j) .
$$

By Lemma 8(ii), for $n=1,2, \ldots, K(\nu)-1$ we have $J_{\nu+n}^{\prime}(j) \leqslant 0$. From the recurrence formula $J_{t-1}(z)-J_{t+1}(z)=2 J_{t}^{\prime}(z)$ we conclude that $0<J_{p+1}(j) \leqslant$ $J_{\nu+3}(j) \leqslant \cdots \leqslant J_{\nu+K(\nu)-1}(j)$ and $0<J_{\nu+2}(j) \leqslant J_{\nu+4}(j) \leqslant \cdots \leqslant J_{\nu+K(\nu)}(j)$. 
Let $M$ be the least odd integer such that $(\nu-\alpha) M \geqslant \alpha \nu$, and let $N$ be the least even integer such that $(\nu-\alpha) N \geqslant \alpha \nu$. Then $N=M \pm 1$. If $N=M+1$, then $M-1<\alpha \nu /(\nu-\alpha)<\frac{1}{2} K(\nu)$. By Lemma 8(iii) we have that $1 \leqslant \frac{1}{2} K(\nu)$ and so $M<\frac{1}{2} K(\nu)+1 \leqslant K(\nu)$. Since $K(\nu)$ is integer valued, $N=M+1 \leqslant K(\nu)$. A similar argument shows that if $N=M-1$, then $N<K(\nu)$ and $M \leqslant K(\nu)$. In either case we have $N, M \leqslant K(\nu)$.

For notational simplicity set $f(n)=(\nu-\alpha) n-\alpha \nu$. Then

$$
\begin{aligned}
\sum_{\substack{n=1 \\
n \text { even }}}^{K(\nu)}((\nu-\alpha) n-\alpha \nu) J_{\nu+n}^{2}(j) & =\sum_{\substack{n=1 \\
n \text { even }}}^{N-2} f(n) J_{\nu+n}^{2}(j)+\sum_{\substack{n=N \\
n \text { even }}}^{K(\nu)} f(n) J_{\nu+n}^{2}(j) \\
& \geqslant J_{\nu+N}^{2}(j) \sum_{\substack{n=1 \\
n \text { even }}}^{K(\nu)} f(n),
\end{aligned}
$$

since $J_{\nu+m}(j) \leqslant J_{\nu+m+2}(j)$ for positive even integers $m<K(\nu)$, and since $f(n)<0$ for $n \leqslant N-2$, and $f(n) \geqslant 0$ for $n \geqslant N$. Hence

$$
\begin{aligned}
\sum_{\substack{n=1 \\
n \text { even }}}^{K(\nu)}((\nu-\alpha) n-\alpha \nu) J_{\nu+n}^{2}(j) \geqslant J_{\nu+N}^{2}(j) \sum_{\substack{n=1 \\
n \text { even }}}^{K(\nu)}((\nu-\alpha) n-\alpha \nu) \\
=\left\{(\nu-\alpha) \frac{K(\nu)}{2}\left(\frac{K(\nu)}{2}+1\right)-\alpha \nu \frac{K(\nu)}{2}\right\} J_{\nu+N}^{2}(j) \\
\geqslant \frac{K(\nu)}{2}\left\{(\nu-\alpha) \frac{K(\nu)}{2}-\alpha \nu\right\} J_{\nu+N}^{2}(j)>0
\end{aligned}
$$

since $2 \alpha \nu /(\nu-\alpha)<K(\nu)$ and $\nu>\alpha$. Similarly,

$$
\begin{aligned}
\sum_{\substack{n=1 \\
n \text { odd }}}^{K(\nu)}((\nu-\alpha) n-\alpha \nu) J_{\nu+n}^{2}(j) & \geqslant J_{\nu+M}^{2}(j) \sum_{\substack{n=1 \\
n \text { odd }}}^{K(\nu)}((\nu-\alpha) n-\alpha \nu) \\
& =\frac{K(\nu)}{2}\left\{(\nu-\alpha) \frac{K(\nu)}{2}-\alpha \nu\right\} J_{\nu+M}^{2}(j)>0 .
\end{aligned}
$$

These, with (19), show that $I(p)>0$ whenever $\nu>\alpha$ and $K(\nu)>2 \alpha \nu /(\nu-\alpha)$. Finally, these conditions are satisfied for all $\nu$ sufficiently large; because $K(\nu) \rightarrow \infty$ as $\nu \rightarrow \infty$ by Lemma $8(i)$, while $2 \alpha \nu /(\nu-\alpha) \rightarrow 2 \alpha$. This completes the proof of Lemma 10.

The estimate contained in Lemma 10 as to how large $\nu$ must be before $I(p)>0$ is probably very crude. Two special cases which will be of use later are $\alpha \leqslant 1.1$ and $\alpha \leqslant 2.411$. We will compute better estimates for $\nu$ in these two cases in the following lemma. 
LEMMA 11. $I(p)>0$ whenever either

(i) $0<\alpha \leqslant 1.1$ and $3.5 \leqslant \nu$; or

(ii) $0<\alpha \leqslant 2.411,20.5 \leqslant \nu$, and $(t+1) j_{t}^{-1}$ is a nondecreasing function on $20.5 \leqslant t$.

Proof. (i) Suppose $\alpha \leqslant 1$.1. If $\nu \geqslant 3.5$ and $n \geqslant 2$, then $(\nu-\alpha) n-\alpha \nu \geqslant$ $(\nu-1.1) 2-1.1 \nu \geqslant 0$. By Lemma 8(iv) $K(\nu) \geqslant 3$ so that by Lemma 8(ii) we have $J_{v+2}^{\prime}(j) \leqslant 0$. From the recurrence relation $J_{v+1}(j)-J_{v+3}(j)=2 J_{v+2}^{\prime}(j)$ we obtain $0<J_{\nu+1}(j) \leqslant J_{\nu+3}(j)$. By Lemma 9 ,

$$
\begin{aligned}
I(p) & \geqslant((\nu-\alpha)-\alpha \nu) J_{\nu+1}^{2}(j)+((\nu-\alpha) 3-\alpha \nu) J_{\nu+3}^{2}(j) \\
& \geqslant(-0.1 \nu-1.1) J_{\nu+1}^{2}(j)+(1.9 \nu-3.3) J_{\nu+3}^{2}(j) \\
& \geqslant(1.8 \nu-4.4) J_{\nu+1}^{2}(j)>0 .
\end{aligned}
$$

This completes the proof of (i).

(ii) Now suppose $\alpha \leqslant 2.411$ and $20.5 \leqslant \nu$. If $n \geqslant 5$, then $(\nu-\alpha) n-\alpha \nu \geqslant$ $5(\nu-2.411)-2.411 \nu \geqslant 2.589 \nu-12.055>0$. By Lemma $8(v), K(\nu) \geqslant 4$ and so by Lemma 8 (ii) $J_{v+1}^{\prime}(j) \leqslant 0, J_{v+2}^{\prime}(j) \leqslant 0$, and $J_{v+3}^{\prime}(j) \leqslant 0$. Using the recurrence formula $J_{t+1}(x)-J_{t+3}(x)=2 J_{t+2}^{\prime}(x)$, we conclude that

$$
\begin{aligned}
& 0=J_{\nu}(j) \leqslant J_{\nu+2}(j) \leqslant J_{\nu+4}(j), \\
& 0<J_{\nu+1}(j) \leqslant J_{\nu+3}(j) .
\end{aligned}
$$

From the recurrence formula $J_{t-1}(x)+J_{t+1}(x)=2 t x^{-1} J_{t}(x)$, we obtain that $J_{\nu+2}(j)=2(\nu+1) j^{-1} J_{\nu+1}(j)$. Hence, by Lemma 9,

$$
\begin{aligned}
I(p)> & (-1.411 \nu-2.411) J_{\nu+1}^{2}(j)+(-0.411 \nu-4.822) J_{\nu+2}^{2}(j) \\
& +(0.589 \nu-7.233) J_{\nu+3}^{2}(j)+(1.589 \nu-9.644) J_{\nu+4}^{2}(j) \\
> & \left\{-0.822 \nu-9.644+(1.178 \nu-14.466) 4(\nu+1)^{2} j^{-2}\right\} J_{\nu+1}^{2}(j) .
\end{aligned}
$$

Let $E(\nu)$ denote the coefficient of $J_{\nu+1}^{2}(j)$ in the last line. Since $(\nu+1) j_{\nu}^{-1}$ is nondecreasing we have $(\nu+1) j^{-1} \geqslant 21.5\left(j_{20.5}\right)^{-1}>0.828335$, where the value of $j_{20.5}$ is found on page 28 of [12]. Hence,

$$
E(\nu)>-0.822 \nu-9.644+(1.178 \nu-14.466)(2.744555) .
$$

A short calculation shows that the right hand side of the inequality in (20) is positive whenever $\nu \geqslant 20.5$. This completes the proof of (ii).

\section{The Rayleigh quotient}

LemMA 12. Let $0<p_{0}<\alpha^{-1}, A(p)=1-\alpha p, \nu(p)=p^{-1}-\alpha$. Let $\lambda_{\mathrm{l}}(p, a)$ and $y_{1}(p, a, x)$ be as defined in Lemma 3, except that $t$ there is replaced by $\nu$ here. 
Let $I(p)$ be as defined by the integral in Lemma 9. Let $I\left(p_{0}\right)>0$, and

$$
I(q, p, a)=\int_{a}^{1}\left\{A^{\prime}(q)-A(q) \ln x\right\} x^{2 q-1} y_{1}^{2}(p, a, x) d x .
$$

Then there are $a_{0} \in(0,1)$ and a neighborhood $D_{0} \subset\left(0, \alpha^{-1}\right)$ of $p_{0}$ such that $I(q, p, a)>0$ whenever $0<a \leqslant a_{0}$ and $q, p \in D_{0}$.

Proof. Fix $b$ and $c$ such that $0<b<p_{0}<c<\alpha^{-!}$. Then

$$
A^{\prime}(q)-A(q) \ln x \geqslant-\alpha-(1-\alpha c) \ln x>0
$$

if $0<x<e^{-\alpha /(1-\alpha c)}, q \in[b, c]$ and $x \in(0,1]$. Hence there is an $a_{1} \in(0,1)$, independent of $q$, such that $A^{\prime}(q)-A(q) \ln x>0$ for $b \leqslant q \leqslant c$ and $0<x \leqslant a_{1}$. Writing $A_{0}, A_{0}^{\prime}$, and $\nu_{0}$ for $A\left(p_{0}\right), A^{\prime}\left(p_{0}\right)$, and $\nu\left(p_{0}\right)$, we have, by definition of $I\left(p_{0}\right)$,

$$
\int_{a}^{1}\left(A_{0}^{\prime}-A_{0} \ln x\right) x^{2 p_{0}-1} J_{\nu_{0}}^{2}\left(j_{\nu_{0}} x^{p_{0}}\right) d x \rightarrow I\left(p_{0}\right) / p_{0} j_{\nu_{0}}^{2}
$$

as $a \rightarrow 0+$; so that this integral is positive for all $a$ sufficiently small, say $0<a \leqslant a_{2}<1$ where $a_{2}$ is independent of $q$ since the whole integral is. Now set $a_{3}=\min \left\{a_{1}, a_{2}\right\} \in(0,1)$. Then

$$
A^{\prime}(q)-A(q) \ln x>0
$$

for $b \leqslant q \leqslant c$ and $0<x \leqslant a_{3}$. Also

$$
H=\int_{a_{3}}^{1}\left(A_{0}^{\prime}-A_{0} \ln x\right) x^{2 p_{0}-1} J_{\nu_{0}}^{2}\left(j_{\nu_{0}} x^{p_{0}}\right) d x>0 .
$$

By Lemma 6, $y_{1}^{2}(p, a, x) \rightarrow J_{\nu}^{2}\left(j_{\nu} x^{p}\right)$ as $a \rightarrow 0+$ uniformly for $b \leqslant p \leqslant c$ and $a_{3} \leqslant x \leqslant 1$. Moreover, $\left[A^{\prime}(q)-A(q) \ln x\right] x^{2 q-1}$ is continuous on $b \leqslant q \leqslant c$, $a_{3} \leqslant x \leqslant 1$, and independent of $p$ and $a$. Consequently

$$
\left\{A^{\prime}(q)-A(q) \ln x\right\} x^{2 q-1} y_{1}^{2}(p, a, x) \rightarrow\left\{A^{\prime}(q)-A(q) \ln x\right\} x^{2 q-1} J_{\nu}^{2}\left(j_{\nu} x^{p}\right)
$$

as $a \rightarrow 0+$ uniformly for $b \leqslant q \leqslant c, b \leqslant p \leqslant c$, and $a_{3} \leqslant x \leqslant 1$. Thus

$$
\begin{aligned}
\int_{a_{3}}^{1}\left\{A^{\prime}(q)-\right. & A(q) \ln x\} x^{2 q-1} y_{1}^{2}(p, a, x) d x \\
& \rightarrow \int_{a_{1}}^{1}\left\{A^{\prime}(q)-A(q) \ln x\right\} x^{2 q-1} J_{\nu}^{2}\left(j_{\nu} x^{p}\right) d x
\end{aligned}
$$

as $a \rightarrow 0+$ uniformly for $b \leqslant q \leqslant c$ and $b \leqslant p \leqslant c$.

The right side of (23) is a continuous function of $(q, p)$ in $[b, c] \times[b, c]$ and by (22) has the positive value $H$ when $q=p=p_{0}$. Therefore, there is a compact neighborhood $D_{0} \subset\left(0, \alpha^{-1}\right)$ of $p_{0}$ such that the right hand side of (23) is greater than $\frac{1}{2} H$ on $D_{0} \times D_{0}$. By the uniform convergence, the left side of (23) exceeds 
$\frac{1}{4} H$ for all $a$ sufficiently small, say $0<a \leqslant a_{4}$. The integrand on the left of (23) is nonnegative on $0<x \leqslant a_{3}$ if $b \leqslant q \leqslant c$ and $b \leqslant p \leqslant c$, by (21). Writing $a_{0}=$ $\min \left\{a_{3}, a_{4}\right\}$,

$$
\int_{a}^{1}\left\{A^{\prime}(q)-A(q) \ln x\right\} x^{2 q-1} y_{1}^{2}(p, a, x) d x>\frac{1}{4} H>0
$$

if $(q, p) \in D_{0} \times D_{0}$ and $0<a \leqslant a_{0}$. This completes the proof.

Definitions. For each $a \in(0,1)$ let $V(a)$ denote the set of all functions $y \in$ $C^{2}[a, 1]$ satisfying $y(a)=0=y(1)$. Let $D$ be any closed subinterval of $\left(0, \alpha^{-1}\right)$ and let $W(a, D)$ denote the set of all functions $y \in V(a)$ such that

$$
\int_{a}^{1}\left\{A^{\prime}(q)-A(q) \ln x\right\} x^{2 q-1} y^{2}(x) d x>0
$$

for all $q \in D$.

LEMMA 13. If $0<p<\alpha^{-1}, A(p)=1-\alpha p, 0<a<1, D$ is any closed subinterval of $\left(0, \alpha^{-1}\right)$, and $y \in W(a, D)$, then

$$
R[p, y, a]=\frac{\int_{a}^{1}\left\{\left(-x y^{\prime}\right)^{\prime}+A^{2}(p) x^{-1} y\right\} y d x}{\int_{a}^{1} x^{2 p-1} y^{2} d x}
$$

is an increasing function of $p$ on $D$.

Proof. Whenever $p>q$ we have $x^{2 p-1}<x^{2 q-1}$ for every $x \in(0,1)$. Hence, if $a \in\left(0, a_{0}\right)$,

$$
\frac{\int_{a}^{1}\left(-x y^{\prime}\right)^{\prime} y d x}{\int_{a}^{1} x^{2 p-1} y^{2} d x} \geqslant \frac{\int_{a}^{1}\left(-x y^{\prime}\right)^{\prime} y d x}{\int_{a}^{1} x^{2 q-1} y^{2} d x}
$$

since $\int_{a}^{1}-\left(x y^{\prime}\right)^{\prime} y d x=\int_{a}^{1} x y^{\prime 2} d x \geqslant 0$. This shows that one term of $R[p, y, a]$ is nondecreasing. For the other, it suffices to show that

$$
g(p)=A^{2}(p) / \int_{a}^{1} x^{2 p-1} y^{2} d x
$$

is an increasing function on $D$. A short calculation shows that $g^{\prime}(p)$ is a quotient with positive denominator and numerator

$$
2 A(p) \int_{a}^{1}\left\{A^{\prime}(p)-A(p) \ln x\right\} x^{2 p-1} y^{2} d x .
$$

Since $y \in W(a, D)$ we have $g^{\prime}(p)>0$ on $D$. The desired result follows directly. 
LEMMA 14. If the conditions of Lemma 12 hold, including $I\left(p_{0}\right)>0$, and $D_{0}$ is the neighborhood of $p_{0}$ constructed there, then $p j_{\nu(p)}$ is a nondecreasing function of $p$ on $D_{0}$.

Proof. Let $p, q \in D_{0}$ and $q<p$. By Lemma 12, $y_{1}(r, a, x) \in W\left(a, D_{0}\right)$ for every $r \in D_{0}$ and $a \in\left(0, a_{0}\right)$. Also, by Rayleigh theory, $\lambda_{1}(r, a)=R\left[r, y_{1}, a\right]$. So by Lemma 13 , since $W\left(a, D_{0}\right) \subset V(a)$,

$$
\lambda_{1}(q, a)=\min _{\substack{W \in W\left(a, D_{0}\right) \\ y \neq 0}} R[q, y, a] \leqslant \min _{\substack{y \in W\left(a, D_{0}\right) \\ y \neq 0}} R[p, y, a]=\lambda_{1}(p, a)
$$

for each $a \in\left(0, a_{0}\right)$. By Lemma $5, \lambda_{1}(r, a) \rightarrow\left(r j_{v(r)}\right)^{2}$ as $a \rightarrow 0+$, so that

$$
\left(q j_{\nu(q)}\right)^{2}=\lim _{a \rightarrow 0+} \lambda_{1}(q, a) \leqslant \lim _{a \rightarrow 0+} \lambda_{1}(p, a)=\left(p j_{\nu(p)}\right)^{2} \text {. }
$$

The desired result now follows immediately.

\section{Main result}

THEOREM 15. The quantity $j_{\nu} /(\nu+\alpha)$ is a strictly decreasing function of $\nu$, for fixed $\alpha$, whenever $\alpha \geqslant 0$ and any of the following hold:

(i) $\alpha \leqslant 1.1$ and $3.5 \leqslant \nu$,

(ii) $\alpha \leqslant 2.411$ and $20.5 \leqslant \nu$,

(iii) $\nu>\alpha$ and $K(\nu)>2 \alpha \nu /(\nu-\alpha)$.

Moreover, $j_{\nu} /(\nu+\alpha) \rightarrow 1$ as $\nu \rightarrow \infty$.

Proof. The case $\alpha=0$ is Theorem 3 of [8]. Let $p=(\nu+\alpha)^{-1}$, so that $\nu=\nu(p)=p^{-1}-\alpha$.

(i) Suppose that $0<\alpha \leqslant 1.1$ and $3.5 \leqslant \nu$. By Lemma $11(\mathrm{i}), I(p)>0$; and by Lemma 14, $p j_{\nu(p)}$ is nondecreasing. By the definition of $p$ and $\nu(p)$ we have that $j_{\nu(p)} /(\nu(p)+\alpha)$ is a nondecreasing function of $p$. Since $p$ is a decreasing function of $\nu, j_{\nu} /(\nu+\alpha)$ is a nonincreasing function of $\nu$. In particular, $j_{\nu} /(\nu+1)$ is nonincreasing for $3.5 \leqslant \nu$.

(ii) By Lemma 11(ii), $I(p)>0$ for $0<\alpha \leqslant 2.411$ and $20.5 \leqslant \nu$. An argument analogous to that above shows that $j_{\nu} /(\nu+\alpha)$ is nonincreasing under these conditions.

(iii) Similarly, under conditions (iii), Lemmas 10 and 14 give that $j_{\nu} /(\nu+\alpha)$ is a nonincreasing function of $\nu$.

(iv) We will now show that under conditions (i), (ii) or (iii), $j_{\nu} /(\nu+\alpha)$ is not only nonincreasing but is indeed a strictly decreasing function of $\nu$. Suppose that it is nonincreasing for $\alpha \leqslant \gamma$ and $\delta \leqslant \nu$. Let $\beta \in(\alpha, \gamma)$. Then $j_{\nu} /(\nu+\beta)$ is 
nonincreasing and $(\nu+\beta) /(\nu+\alpha)$ is strictly decreasing for $\delta \leqslant \nu$. Therefore $j_{\nu} /(\nu+\alpha)$ is strictly decreasing, since

$$
\frac{j_{\nu}}{\nu+\alpha}=\frac{j_{\nu}}{\nu+\beta} \cdot \frac{\nu+\beta}{\nu+\alpha}
$$

(v) From the asymptotic expansion $j_{\nu}=\nu+O\left(\nu^{1 / 3}\right)$ as $\nu \rightarrow \infty$, found in [14] or on page 371 of [1], it follows immediately that $j_{v} /(\nu+\alpha) \rightarrow 1$. This completes the proof of Theorem 15.

\section{Consequences}

Theorem 15 can be used to compute upper bounds for $j_{\nu}$. For example, using (ii) we have

$$
\begin{aligned}
j_{\nu} & <j_{20.5}(\nu+2.411)(22.911)^{-1} \\
& <1.133(\nu+2.411) \\
& <1.133 \nu+2.732
\end{aligned}
$$

for $20.5<\nu$, where the value of $j_{20.5}$ has been taken from page 28 of [15]. To the authors' knowledge the best upper bound for $j_{\nu}$ previously known is

$$
j_{\nu}<\left\{\frac{4}{3}(\nu+1)(\nu+5)\right\}^{1 / 2}
$$

which may be found on page 487 of [15]. A short calculation shows that the upper bound in (24) is better than that in (25) for $20.5<\nu$.

The theorem may also be used to compute a lower bound for $j_{\nu}$. Since $j_{0}<2.405$ and $j_{\nu}(\nu+\alpha)^{-1}>1$ whenever $\alpha \leqslant 2.411$ and $20.5 \leqslant \nu$, we have $j_{\nu}>\nu$ $+j_{0}$ whenever $20.5 \leqslant \nu$. Using the tables in [12], [5, page 167], and [3, page 317], we find that

$$
\begin{array}{cc}
j_{\nu}>20.5+j_{0} \geqslant \nu+j_{0} & \text { for } \nu \in[18,20.5], \\
j_{\nu}>18+j_{0} \geqslant \nu+j_{0} & \text { for } \nu \in[15.5,18], \\
j_{\nu}>15.5+j_{0} \geqslant \nu+j_{0} & \text { for } \nu \in[13.5,15.5], \\
\cdots \ldots \ldots \ldots & \cdots \cdots \ldots \ldots \ldots \\
j_{\nu}>0.15+j_{0} \geqslant \nu+j_{0} & \text { for } \nu \in[0.1,0.15], \\
j_{\nu}>0.1+j_{0} \geqslant \nu+j_{0} & \text { for } \nu \in[0.075,0.1], \\
j_{\nu}>0.075+j_{0} \geqslant \nu+j_{0} & \text { for } \nu \in[0.05,0.075] .
\end{array}
$$

Hence, $j_{v}>\nu+j_{0}$ whenever $0.05<\nu$. 
On page 508 of [15] (where $j_{\nu}$ is denoted by $j$ ) it is shown that, for $\nu>0$,

$$
\frac{d j}{d \nu}=\frac{2 \nu}{j J_{\nu+1}^{2}(j)} \int_{0}^{j} s^{-1} J_{\nu}^{2}(s) d s .
$$

Using the identity

$$
2 \nu \int_{0}^{z} s^{-1} J_{\nu}^{2}(s) d s=J_{\nu}^{2}(z)+2 \sum_{m=1}^{\infty} J_{\nu+m}^{2}(z)
$$

which is (5) on page 152 of [15], we have

$$
\frac{d j}{d \nu}>\frac{2}{j}\left\{1+\frac{J_{\nu+2}^{2}(j)}{J_{\nu+1}^{2}(j)}\right\} .
$$

From the recurrence formula $J_{t-1}(x)+J_{t+1}(x)=2 t x^{-1} J_{t}(x)$, with $t=\nu+1$, we have $J_{\nu+2}(j)=2(\nu+1) j^{-1} J_{\nu+1}(j)$. Hence,

$$
\frac{d j_{\nu}}{d \nu}>2 j_{\nu}^{-1}\left\{1+4(\nu+1)^{2} j_{\nu}^{-2}\right\} \text {. }
$$

From the table on page 317 of [3] we find that $j_{\nu}<2.4817$ on the interval $[0,0.05]$. Hence, on this interval,

$$
\frac{d j_{\nu}}{d \nu}>2(2.4817)^{-1}\left\{1+4(2.4817)^{-2}\right\}>1 \text {. }
$$

It follows directly that $j_{\nu}>\nu+j_{0}$ for $\nu \in(0,0.05]$. Combining the above calculations, we have

THEOREM 16. If $j_{\nu}$ is the least positive zero of $J_{v}$, then

$$
j_{\nu}>\nu+j_{0} \text { for } \nu>0 \text {. }
$$

The most commonly cited lower bound for $j_{\nu}$, other than $j_{\nu}>\nu$, seems to be

$$
j_{\nu}>\{\nu(\nu+2)\}^{1 / 2}
$$

which may be found on page 486 of [15]. Clearly $\nu+j_{0}>\{\nu(\nu+2)\}^{1 / 2}$ for all $\nu>0$. In [9] it is shown that

$$
j_{v} \geqslant\left(\nu^{2}+j_{0}^{2}\right)^{1 / 2}
$$

Again it is clear that, for $\nu>0, \nu+j_{0}>\left(\nu^{2}+j_{0}^{2}\right)^{1 / 2}$.

In [4] it is shown that

$$
j_{\nu} \geqslant \nu \pi / 2+3 \pi / 4
$$

whenever $\nu \in\left[0, \frac{1}{2}\right]$. The inequality in Theorem 16 becomes equality when $\nu=0$, while the expression in (26) has a strict inequality. However, when $\nu=\frac{1}{2}$ equality holds in (26). Hence, for $\nu \in\left[0, \frac{1}{2}\right]$ neither of the inequalities in Theorem 16 or 
(26) implies the other. It should be emphasized that Theorem 16 is valid for all $\nu>0$ while (26) is valid only for $\nu \in\left[0, \frac{1}{2}\right]$.

The inequality in Theorem 16 may be interpreted as follows. Consider a membrane, clamped along its edges, in the form of a circular sector $0 \leqslant r \leqslant 1$, $0 \leqslant \theta \leqslant \alpha$, where $\alpha<2 \pi$. The fundamental frequency for the transverse vibrations of the membrane is proportional to $j_{\pi / \alpha}$. From Theorem 16 we see that the fundamental frequency is a superlinear function of $\alpha^{-1}$.

It is of interest to consider the behavior of the function $f(\nu)=j_{v} /\left(\nu+j_{0}\right)$. Using the tables in [12] for $\nu=\frac{1}{2} k, k=0,1, \ldots, 41$, we find that $f\left(\frac{1}{2} k\right)$ increases from $f(0)=1.0000$ to $f(4.5)=1.1850$ and then decreases to $f(20.5)=1.1332$. $^{*}$

\section{References}

[1] M. Abramowitz and I. A. Stegun (eds.), Handbook of mathematical functions (Dover, New York, 1970).

[2] R. Courant and D. Hilbert, Methods of mathematical physics, Vol. I (Interscience, New York, 1953).

[3] A. Gray, G. B. Mathews and T. M. MacRobert, $A$ treatise on the theory of Bessel functions (2nd edition, Dover, New York, 1966).

[4] H. W. Hethcote, "Error bounds for asymptotic approximations to zeros of transcendental functions", SIAM J. Math. Anal. I (1970), 147-152.

[5] E. Jahnke and F. Emde, Tables of functions (4th edition, Dover, New York, 1945).

[6] J. T. Lewis and M. E. Muldoon, "Monotonicity and convexity properties of zeros of Bessel functions”, SIAM J. Math. Anal. 8 (1977), 171-178.

[7] Y. L. Luke, Integrals of Bessel functions (McGraw-Hill, New York, 1962).

[8] R. C. McCann, "Inequalities for the zeros of Bessel functions", SIAM J. Math. Anal. 8 (1977), $166-170$.

[9] R. C. McCann, "Lower bounds for the zeros of Bessel functions", Proc. Amer. Math. Soc. 64 (1977), 101-103.

[10] S. G. Mikhlin, Variational methods of mathematical physics (Macmillan, New York, 1964).

[11] National Bureau of Standards, Tables of spherical Bessel functions, Vol. II (Columbia University Press, New York, 1950).

[12] F. W. J. Olver, (ed.), Royal society mathematical tables, Vol. 7, Bessel functions, Part III, Zeros and associated values (Cambridge University Press, Cambridge, 1960).

[13] H. L. Royden, Real analysis (Macmillan, New York, 1963).

[14] F. G. Tricomi, "Sulle funzioni di Bessel di ordine e argomento pressochè uguale", Atti. Accad. Sci. Torino Cl. Sci. Fis. Mat. Nat. 83 (1949), 3-20.

[15] G. N. Watson, A treatise on the theory of Bessel functions (Cambridge University Press, 2nd edition, Cambridge, 1945).

*Theorem 15 shows that $f(\nu)$ thereafter decreases towards 1 ; and the range of this function illuminates Theorem 16. 